\title{
Single-base resolution methylomes of upland cotton (Gossypium hirsutum L.) reveal epigenome modifications in response to drought stress
}

Xuke Lu ${ }^{1,2}$, Xiaoge Wang ${ }^{1}$, Xiugui Chen ${ }^{1}$, Na Shu', Junjuan Wang ${ }^{1}$, Delong Wang ${ }^{1}$, Shuai Wang ${ }^{1}$, Weili Fan', Lixue Guo ${ }^{1}$, Xiaoning Guo ${ }^{1}$ and Wuwei Ye ${ }^{1 *}$

\begin{abstract}
Background: DNA methylation, with a cryptic role in genome stability, gene transcription and expression, is involved in the drought response process in plants, but the complex regulatory mechanism is still largely unknown.

Results: Here, we performed whole-genome bisulfite sequencing (WGBS) and identified long non-coding RNAs on cotton leaves under drought stress and re-watering treatments. We obtained 31,223 and 30,997 differentially methylated regions (representing $2.48 \%$ of the genome) after drought stress and re-watering treatments, respectively. Our data also showed that three sequence contexts, including ${ }^{\mathrm{m}} \mathrm{CpG},{ }^{\mathrm{m}} \mathrm{CHG},{ }^{\mathrm{m}} \mathrm{CHH}$, all presented a hyper-methylation pattern under drought stress and were nearly restored to normal levels after the re-watering treatment. Among all the methylation variations, asymmetric $\mathrm{CHH}$ methylation was the most consistent with external environments, suggesting that methylation/ demethylation in a $\mathrm{CHH}$ context may constitute a novel epigenetic modification in response to drought stress. Combined with the targets of long non-coding RNAs, we found that long non-coding RNAs may mediate variations in methylation patterns by splicing into microRNAs. Furthermore, the many hormone-related genes with methylation variations suggested that plant hormones might be a potential mechanism in the drought response.
\end{abstract}

Conclusions: Future crop-improvement strategies may benefit by taking into account not only the DNA genetic variations in cotton varieties but also the epigenetic modifications of the genome.

Keywords: Methylomes, Upland cotton, Epigenome modifications, Long non-coding RNAs, Drought stress

\section{Background}

An increasing number of documents have evidenced the importance of DNA methylation in the process of plant growth and development, plant defense, and the response to adversity stress, including salt, drought and heavy metal stress. Naturally occurring modifications in a single gene locus in plants may yield heritable morphological changes without alteration of the DNA sequence [1,2], and some of these modifications may even be passed down through several generations. Arabidopsis, a model plant, is the first plant for which a whole-genome methylome map was

\footnotetext{
* Correspondence: yew158@163.com

${ }^{1}$ State Key Laboratory of Cotton Biology/Institute of Cotton Research of Chinese Academy of Agricultural Sciences/Key Laboratory for Cotton Genetic Improvement, Anyang 455000, Henan, China

Full list of author information is available at the end of the article
}

deciphered, for its compact genome and rapid cycle [3-5]. Cotton has always been known as a primary model plant for studying genome polyploidization [6], cell fate determination, cell elongation and cell wall formation [7]. Currently, the genomes of diploid cottons G. arboreum (AA) and G. raimondii (DD) and complex allotetraploid G. hirsutum L. (AADD) have all been completed [8-10], and high-quality reference genomes and improving sequencing technologies may better ensure the whole-genome methylome analyses of cotton and some other moderate-sized crop genomes, such as the recent discovery of the tomato fruit methylome and of hypomethylation in the rice endosperm [11, 12]. Here, we investigate whether wholegenome epigenome reprogramming occurs during the 
biologically important processes of cotton in response to drought stress.

The response to drought stress in plants is a complicated process, involving several genes and metabolic networks, and DNA methylation is one of them. Publications have shown that drought can induce alterations of the DNA methylation locus and patterns in plants with variety specificity, tissue specificity and stress specificity [13-15]. The establishment and maintenance of DNA methylation patterns that result in gene expression modulation is one of the key steps in epigenetic regulation during normal growth and developmental programs. Documents about transcriptome analysis and drought-response miRNA identification and functional analysis have been publicated [16-18]. Recent discoveries have identified long non-coding RNAs (lncRNAs, defined as transcripts of $>200 \mathrm{bp}$ without protein-coding capacity) as new important players in DNA methylation regulation [19]. A recent study published in Nature showed a novel lncRNA arising from the CEBPA gene locus (termed ecCEBPA) that is critical for regulating DNA methylation at this site through the binding of ecCEBPA with DNA methyltransferase1, DNMT1 [20]. Interestingly, a robust increase was observed in the levels of DNA methylation of the CEBPA promoter region following the depletion of this non-coding transcript [20]. However, the alterations of the DNA methylome and the possible roles of lncRNAs in regulating the occurrence of methylation are still unclear under drought conditions in cotton.

Here, we provide a high-resolution and high-coverage map comprising the methylation status of individual cytosines throughout the cotton genome in response to drought stress. On the basis of the possible regulatory functions of lncRNAs, and to investigate the roles of lncRNAs in adjusting the epigenome modifications, we integrated the DNA methylome and lncRNAs. Based on this resource, future studies can use low-coverage methylome sequencing to determine the impact of differentially methylated regions on gene expression, chromosome biology, and transgenerational inheritance. For example, this will allow breeders to determine the contribution of epigenetic modifications to phenotypic variations [21], and the breeders can utilize a form of "epigenetic selection" to analogously select the individuals with desired epigenomic patterns in breeding programs.

\section{Results}

\section{Genome-wide patterns of DNA methylation}

Bisulfite treatment of DNA, termed the "gold standard" of DNA methylation research, can convert unmethylated cytosines into uracils while leaving methylated cytosines unchanged, which allows the generation of "methylomes" at single-base resolution [22]. The bisulfite-converted DNA of drought-tolerant cotton variety ZhongH177 under different treatments, including CK, drought (relative water content (RWC) reached approximately $7 \%$ in pots) (Fig. 1a), and re-watering, was sequenced with Illumina $\mathrm{Hi}-\mathrm{Seq}$ instruments at a coverage that provided information on the methylation states of individual cytosines with high confidence. The percentage of methylated cytosines varied depending on the local sequence context $(\mathrm{C}, \mathrm{CG}, \mathrm{CHG}$ and $\mathrm{CHH}$ ) and the external treatments (CK, drought and rewatering). The results of WGBS are listed in Table 1 . We obtained 228,002,142 and 256,948,717 and 253,873,809 reads after CK, drought and re-watering stresses, respectively. In addition, the mapping rate was $65.28,67.68$ and $67.51 \%$, respectively, showing that the method and the results have high reliability and accuracy. The distribution of methylation sites in each chromosome was analyzed (Fig. 1b and Additional file 1: Figure S1, Additional file 2: Figure S2 and Additional file 3: Figure S3). Among the methylated cytosines, we found that more than half of them were located in asymmetric $\mathrm{CHH}$ contexts (Table 1). The total ${ }^{\mathrm{m}} \mathrm{C}$ in the whole genome under three environments (CK, D and re-watering) was 27.99, 32.34 and $29.95 \%$, respectively, showing an up-regulated methylation level after drought stress and a re-down-regulated trend after recovery from drought stress by re-watering compared with CK. The duplication rate (the percentage of repetitive sequences in all clean sequencing reads) increased approximately $2 \%$ after drought stress and re-watering (Table 2), which showed that repetitive sequences may be important in response to drought stress through the alterations of the methylation level and state. Interestingly, we found that methylated cytosines in ${ }^{\mathrm{m}} \mathrm{CpG},{ }^{\mathrm{m}} \mathrm{CHG}$, ${ }^{\mathrm{m}} \mathrm{CHH}$ contexts all showed a hyper-methylation pattern after drought stress compared with $\mathrm{CK}$ and re-watering, but the alterations in the $\mathrm{CHH}$ contexts were more significant than the alterations in other contexts. This finding suggested that methylation levels in asymmetric $\mathrm{CHH}$ contexts were dynamically changing with external environments and were mostly correlated with environments. In symmetric CG and CHG contexts, more than half of these cytosines were methylated while only a small proportion were methylated in the $\mathrm{CHH}$ context (Table 2).

\section{Cytosine DNA methylation may have sequence preference}

Studies showed that the occurrence of cytosine methylation was associated with its nearest sequence context [4]. Therefore, we used a tool named Logo Plots, which explores the sequence information of a methylated site or its nearby regions, to help us to understand the preference of methylated sites. The method is described in the "Methods" section. Among all methylated ${ }^{\mathrm{m}} \mathrm{C}$ sites, the sequence preference around ${ }^{\mathrm{m}} \mathrm{C}$ sites varied with the CG context and non-CG context (Fig. 2a). In the symmetric CG context, ${ }^{\mathrm{m}} \mathrm{C}$ frequently occurred at TCGA sequences, regardless of the presence of low-methylation-level sites or high- 


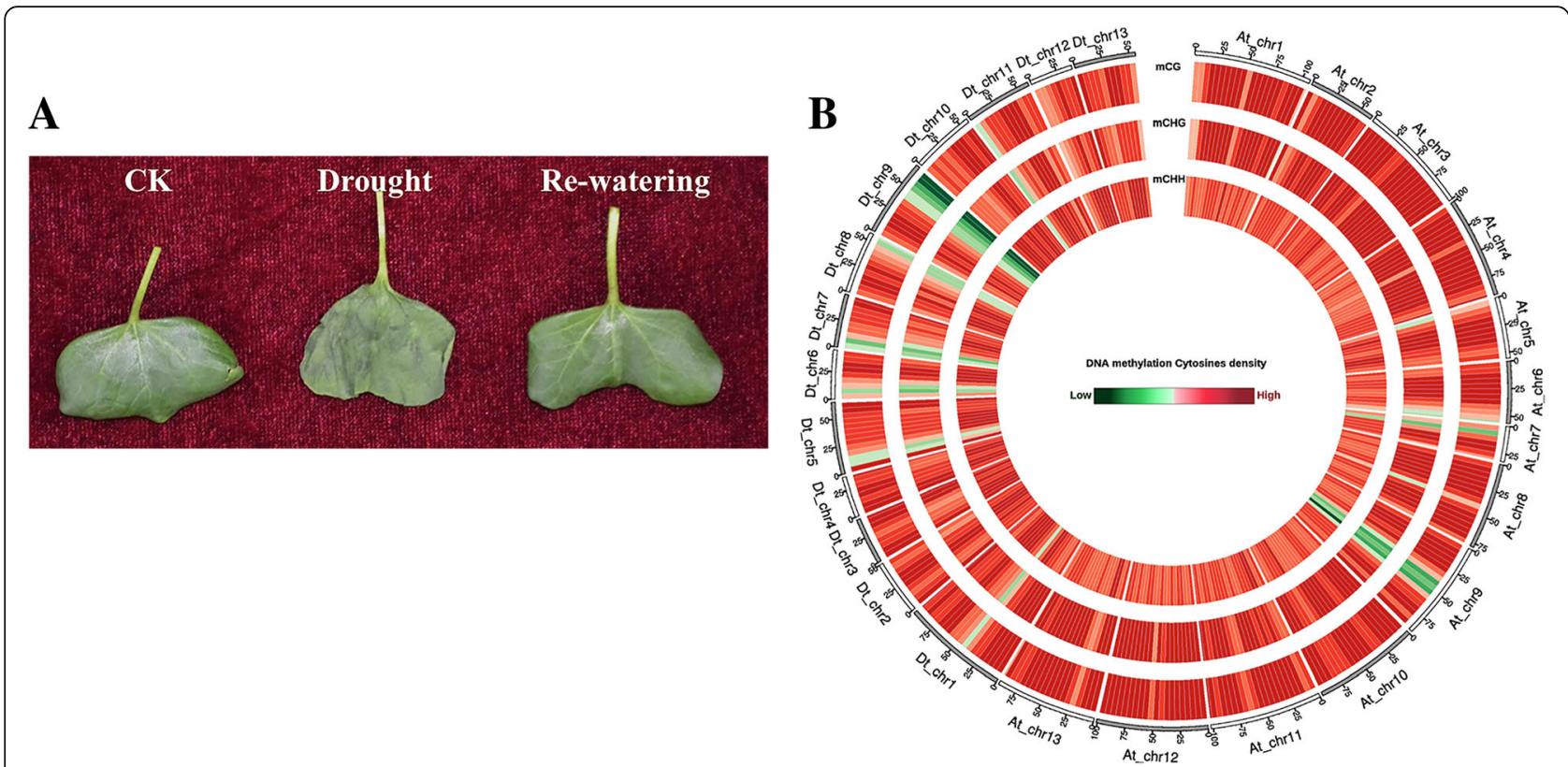

Fig. 1 Epigenome of Gossypium hirsutum L. a Morphological changes of cotyledons under different treatments. b Density plot of 5-methylcytosine in sequence contexts ( $\mathrm{mCG}, \mathrm{mCHG}$ and $\mathrm{mCHH}$ where $\mathrm{mC}$ signifies 5-methylcytosine, and $\mathrm{H}$ represents $\mathrm{A}, \mathrm{C}$ or $\mathrm{T}$ ). The circles from the outside to inside represent $\mathrm{mCG}, \mathrm{mCHG}, \mathrm{mCHH}$, respectively

methylation-level sites. In the CHG context, we found that the ${ }^{\mathrm{m}} \mathrm{C}$ of high methylation levels occurred in CTG sequences, whereas at low methylation levels, ${ }^{\mathrm{m}} \mathrm{C}$ appeared in CTG or CAG sequences, which may be caused by drought stress and re-watering treatments. However, in the $\mathrm{CHH}$ context, low-methylation-level ${ }^{\mathrm{m}} \mathrm{C}$ sites often occurred in the CAA sequence context, and high-methylation-level ${ }^{\mathrm{m}} \mathrm{C}$ sites occurred in the CTA context. Therefore, we inferred that most highmethylation-level ${ }^{\mathrm{m}} \mathrm{C}$ sites regularly occurred in the CTN (N represents A, T, C, G) context, while lowmethylation-level ${ }^{\mathrm{m}} \mathrm{C}$ sites occurred in the CAN (N represents A, T, C, G) context. Studies in humans suggest that CHG cytosine in the TACAG context is more easily methylated, and this type of methylation is always found at splicing sites [23]. Along with the alterations of methylation levels, the frequency of CG dinucleotide and its nearest sequences is also changed. However, this is not always consistent in all creatures. In humans, the methylation extent of the $\mathrm{CpG}$ dinucleotide is not closely related to its nearest sequence context; however, in terms of $\mathrm{CHH}$ and $\mathrm{CHG}, \mathrm{T}$ and $\mathrm{A}$ are always upstream and downstream of cytosines with a higher methylation level [24].

\section{Differentially methylated regions (DMRs) responding to drought stress}

Based on the annotation of gene elements (including promoters, exons and introns) [25], we compared the methylation levels in each context in each gene element (Fig. 2b), which can help to identify the functions of methylation alterations in each element in response to drought stress. We found that promoters displayed significantly higher levels of methylation than did exons and introns, suggesting that the methylation levels of $\mathrm{C}$ may be correlated with gene elements. In each gene element, the frequencies of each context, including CG methylation, $\mathrm{CHG}$ methylation and $\mathrm{CHH}$ methylation, were disparate, and CG methylation accounted for approximately half of the total ${ }^{\mathrm{m}} \mathrm{C}$, which suggested that CG methylation was the most important methylation pattern in the three gene elements. Previous publications

Table 1 Bisulfite sequencing summary

\begin{tabular}{lllcllll}
\hline Samples & Total reads & Mapped reads & Mapping rate (\%) & $\begin{array}{l}{ }^{\mathrm{m}} \mathrm{C} \\
\text { percent (\%) }\end{array}$ & $\begin{array}{l}{ }^{\mathrm{m} C G} \\
\text { percent (\%) }\end{array}$ & $\begin{array}{l}{ }^{\mathrm{m}} \mathrm{CHG} \\
\text { percent (\%) }\end{array}$ & $\begin{array}{l}{ }^{\mathrm{m}} \mathrm{CHH} \\
\text { percent (\%) }\end{array}$ \\
\hline CK & $349,288,166$ & $228,002,142$ & 65.28 & $201,543,688(100)$ & $47,879,971(23.76)$ & $45,874,296(22.76)$ & $107,789,421(53.48)$ \\
D & $379,630,307$ & $256,948,717$ & 67.68 & $232,876,499(100)$ & $50,588,873(21.72)$ & $48,445,561(20.80)$ & $13,3842,065(57.47)$ \\
Re_W & $376,067,028$ & $253,873,809$ & 67.51 & $215,698,912(100)$ & $49,367,000(22.89)$ & $47,336,957(21.95)$ & $118,994,955(55.17)$
\end{tabular}

Total reads are the clean sequencing reads after the data filtration. Mapping rate are the reads with unique positions on reference genome after alignments. ${ }^{\mathrm{m}} \mathrm{C}$, ${ }^{\mathrm{m}} \mathrm{CG},{ }^{\mathrm{m}} \mathrm{CHG},{ }^{\mathrm{m}} \mathrm{CHH}$ percent (\%) are the percentages of methylated ${ }^{\mathrm{m}} \mathrm{C}$ in each context in all methylated cytosines from the whole genome 
Table 2 Statistical results of methylated cytosines in different contexts

\begin{tabular}{llllll}
\hline Samples & Duplication rate (\%) & ${ }^{\mathrm{m}} \mathrm{C}$ percent (\%) & ${ }^{\mathrm{m}} \mathrm{CpG}$ percent (\%) & ${ }^{\mathrm{m}} \mathrm{CHG}$ percent (\%) & ${ }^{\mathrm{m} C H H}$ percent (\%) \\
\hline CK & 10.70 & $27.99 \%$ & $58.42 \%$ & $53.77 \%$ & $19.50 \%$ \\
D & 12.48 & $32.34 \%$ & $61.71 \%$ & $56.78 \%$ & $24.21 \%$ \\
Re_W & 12.24 & $29.95 \%$ & $60.22 \%$ & $55.48 \%$ & $21.52 \%$ \\
\hline
\end{tabular}

Duplication rate is the percentage of repetitive sequences in all clean sequencing reads. ${ }^{\mathrm{m}} \mathrm{C}$ percent $(\%)$ is the percentage of methylated cytosines in all cytosines from the whole genome. ${ }^{\mathrm{m}} \mathrm{CG},{ }^{\mathrm{m}} \mathrm{CHG}$, ${ }^{\mathrm{m}} \mathrm{CHH}$ percent (\%) are the percentages of methylated ${ }^{\mathrm{m}} \mathrm{CG},{ }^{\mathrm{m}} \mathrm{CHG},{ }^{\mathrm{m}} \mathrm{CHH}$ in all corresponding cytosines context (C, $\mathrm{CG}$, $\mathrm{CHG}$, $\mathrm{CHH}$ and $\mathrm{H}$ represents $\mathrm{A}, \mathrm{C}, \mathrm{T}$ ) in genome, respectively

have showed that methylation most frequently occurs in the so-called CpG islands in the 5' regulatory gene regions (promoters) [26]. We then compared the methylation levels of different contexts in promoter, exon and intron pre- and post-drought stress, and minuscule changes were found, except for $\mathrm{CHH}$ methylation (Fig. 2b). Interestingly, we found that the $\mathrm{CHH}$ methylation in the promoter region exhibited an up-regulated pattern under drought stress compared with the control and a slightly down-regulated pattern after the re-watering treatment compared with

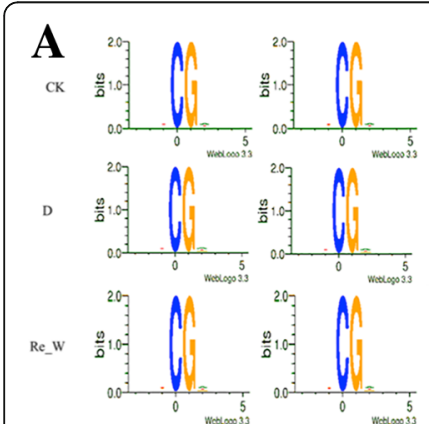

a

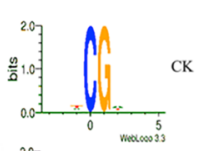

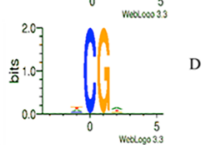

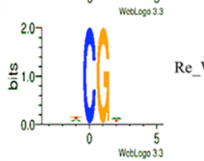

c

(1)

B

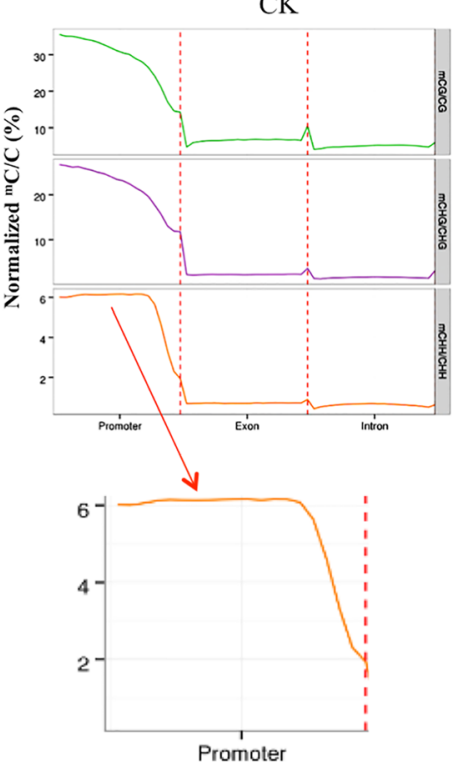

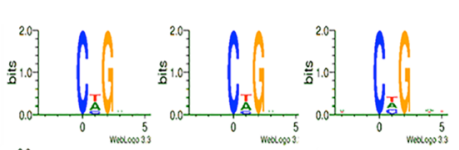

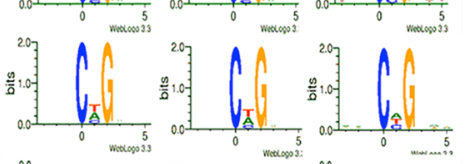

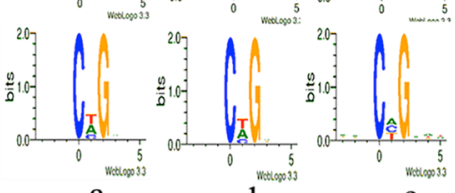

(<smiles>C1CC(C2CC2)C1</smiles>

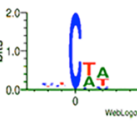<smiles>C1C2CC3CC1C3C2</smiles>

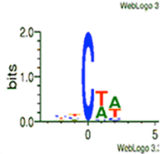

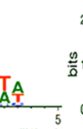<smiles>CC(C)C</smiles>

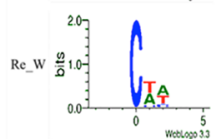

a

c

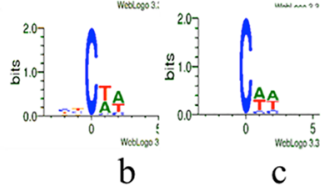

(3)

D
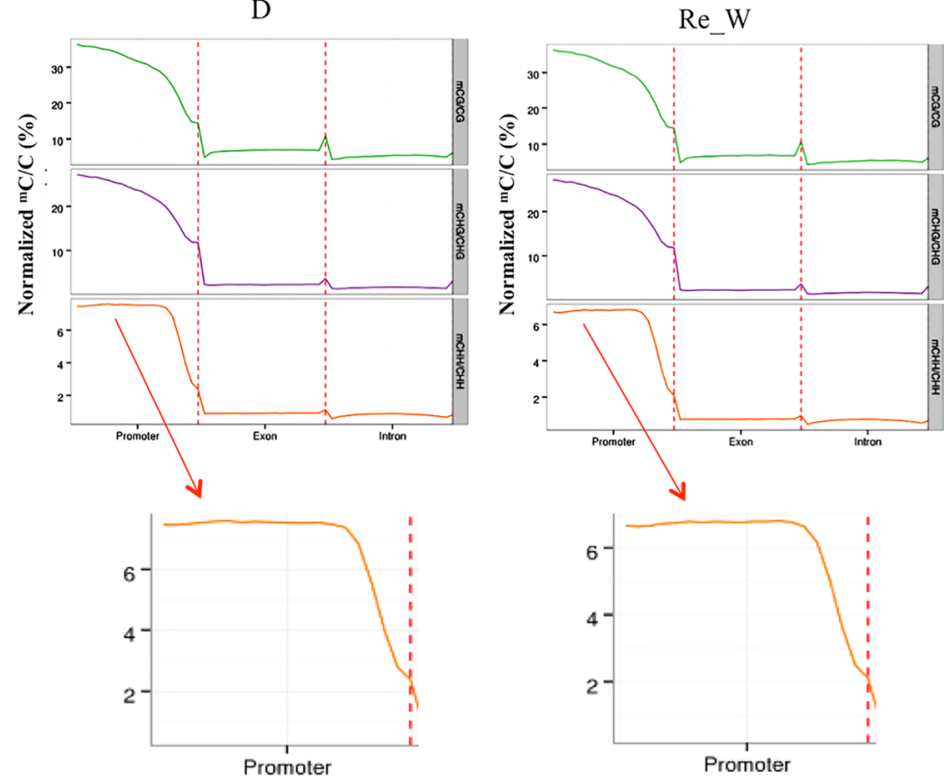

Promoter

Fig. 2 Sequence preference of Cytosine DNA methylation and differentially methylated cytosines in gene elements. (A) Weblogo analysis of bases around ${ }^{5 \mathrm{~m}-} \mathrm{C}$ in different sequence contexts. $\mathrm{X}$ axis represents bases position and $\mathrm{Y}$ axis represents the degree of base enrichment. The position of $\mathrm{C}$ was defined as 0. (1), (2), (3) represent CG, CHG and $\mathrm{CHH}$ context, respectively. a, b, c represent all ${ }^{\mathrm{m}} \mathrm{C}$ sites, high methylation sites and low methylation sites, respectively. In CG context, we defined that methylation level of high methylation level sites was $>75 \%$ and others were low methylation level sites. In non-CG context, we defined that methylation level of high methylation level sites was $>25 \%$ and others were low methylation level sites. (B) Methylation level on different genome elements. $X$ axis represents different gene elements, and $Y$ axis represents methylation levels. Each element of each gene was divided into 20 bins and the average of all bins in each Gene element was obtained. Different colours represent different sequence contexts 
drought stress. This also suggested that the $\mathrm{CHH}$ methylation level is dynamic with environments and that $\mathrm{CHH}$ methylation may be very closely correlated with drought stress. Studies have shown that methylation in the $\mathrm{CHH}$ context increases compared with the CG and CHG context, and this $\mathrm{CHH}$ methylation can be explained by genes promoting de novo methylation on flanking intergenic chromatin (particularly within a few kb of gene starts, that is, always in promoter regions) [27]. Consequently, we inferred that the mechanism through which methylation is altered in promoter regions can be correlated with genes promoting de novo methylation to regulate gene expression in response to drought stress.

Increasing evidence has shown that the methylation and demethylation processes are always correlated with altered gene expression [28]. Therefore, we analyzed the influences of methylation changes on gene body and promoter regions, and the results are shown in (Fig. 3a). To analyze the relationship between DNA methylation and gene expression, the FPKM-log_ratio was used to uniformize the FPKM value of genes and to avoid a large range of gene expression levels. The results indicated that the range of methylation changes in the gene body region was large, while the promoter was small. Furthermore, the overall methylation level in the promoter was higher than that in the gene body region, which may be closely related to the gene functions. Combined with methylation variations and gene expression changes in the gene body and promoter regions, we found that gene expression could be influenced by DNA methylation changes. In the gene body region, the range of gene expression alterations was narrow compared with those of promoters, and there were some additional genes with a great difference in expression in the promoter region, which indicated that methylation in the gene body may influence gene expression levels to a relatively low and intensive level, while a higher methylation level in a promoter may yield a scattered scope of gene expression. Taken together, our results showed that methylation in the gene body and promoter region can suppress gene expression to some extent.

To assess the role of methylation in response to drought stress, we examined differentially methylated regions (DMRs) under drought stress compared with controls, which were always considered to participate in the process of gene regulation. We discovered 31,223 DMRs under drought stress compared with the control, and the number of DMRs decreased to 30,997 after the re-watering treatment (Fig. 3b), which indicated that some DMRs were dynamically changed along with the external environments and that, when the adversity was removed, the methylation sites would return to the normal pattern. We also

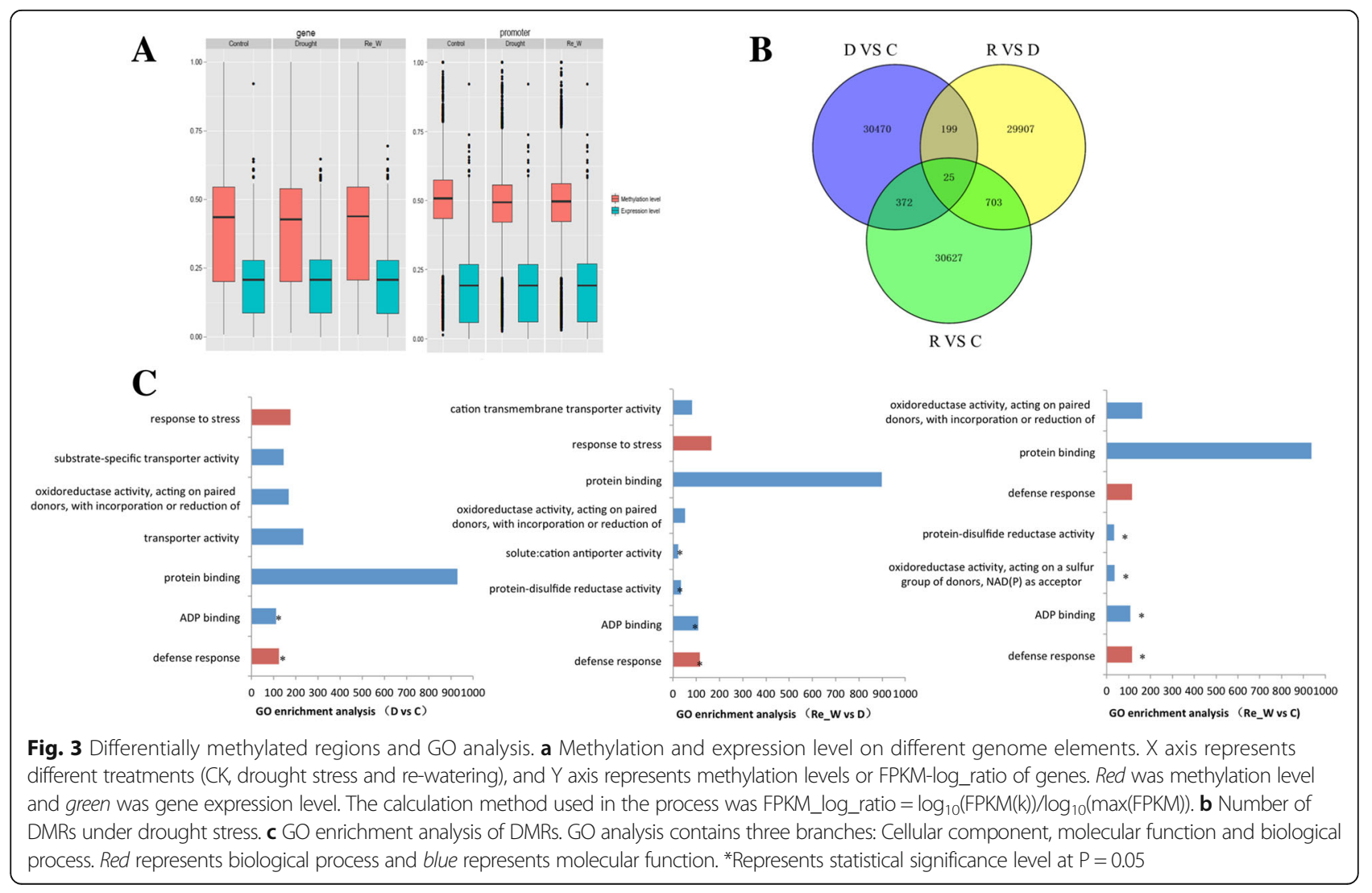


performed a DMR length analysis in each chromosome (Additional file 4: Figure S4, Additional file 5: Figure S5 and Additional file 6: Figure S6) and found that the length of DMRs varied among 26 chromosomes, which may be correlated with the gene numbers, transposon numbers and certain other elements in each chromosome. To determine the role of DMRs during the response to drought stress, we performed Gene Ontology term enrichment analysis of differentially methylated genes (Fig. 3c). Genes involved in protein binding, ADP binding, defense response and transporter activity were highly enriched. Moreover, a GO analysis of hyper- and hypo- methylated genes showed that these genes with great changes in methylation levels or methylation patterns may be closely correlated with drought (Additional file 7: Figure S7 and Additional file 8: Figure S8), in turn suggesting that methylation alterations may be a mechanism to regulate gene expression in response to drought stress. The KEGG pathway analysis results showed that pathways such as ribosomes, RNA degradation, protein processing in the endoplasmic reticulum, and plant hormone signal transduction may be closely correlated with the response to drought (Additional file 9: Figure S9). Above all, we inferred that plants can change the methylation levels or patterns of certain genes in some pathways related to stress to regulate gene expression, which is a mechanism to respond to stress.

\section{Long non-coding RNAs (IncRNAs) may mediate the occurrence of DNA methylation}

DNA methylation was the first discovered epigenetic mechanism [29, 30] and was reported to be regulated by IncRNAs [31]. To assess the role of lncRNAs in regulating DNA methylation changes in response to drought stress, we performed whole-genome screening and identification of lncRNAs responding to drought, and we obtained 9,989 lincRNAs, 678 anti-sense lncRNAs and 153 intronic lncRNAs through five strict filtration steps (Fig. 4a). The regulatory potential of lncRNAs has never ceased to amaze: from RNA catalysis to RNA-mediated splicing, RNA-based silencing, and RNA-directed DNA methylation [32]. LncRNAs are always the precursors of microRNAs and play a role by breaking into one or more microRNAs. Therefore, we conducted microRNA prediction of these identified lncRNAs with a threshold (E-value1 $\leq 0.05)$ (Fig. 4b), and the results indicated that approximately $40 \%$ of lncRNAs were the precursors of microRNAs.

In plants, DNA methylation typically occurs by RNAdirected DNA methylation ( $\mathrm{RdDM}$ ), which directs transcriptional gene silencing of transposons and endogenous transgenes, and RdDM is driven by non-coding RNAs (ncRNAs) [33]. Publications have reported that de novo DNA methylation can be directed by many long non- coding RNAs (80-nucleotide) and small interfering RNAs (24-nucleotide) [34, 35]. We also performed repetitive sequence and transposon analyses of lncRNAs (Fig. 4c), as many 20-24 nucleotide siRNAs were derived from repetitive sequences and transposons. Consequently, we speculated that it may be a regulatory mechanism through which long non-coding RNAs mediate the occurrence by splicing into microRNAs during the drought response process. Based on lncRNA-seq data, we screened the DMRs with a corrected $p$-value $\geq 0.05$, and 514 target genes were identified with high confidence. The GO analysis of these genes is presented in Fig. 4d.

\section{DNA methylation may participate in the regulation of plant hormones}

Based on the analysis of GO and KEGG pathways, we discovered 65 differentially methylated genes associated with hormones (Fig. 5a), of which, 6, 34, 9 and 16 were related to ethylene, auxin, gibberellin and cytokinin, respectively. To further investigate whether DNA methylation is involved in the regulation of hormone-related genes, we used methylation-specific PCR (MSP) to test the methylation changes pre- and post-drought treatment. Nearly $70.76 \%(46 / 65)$ of genes were induced to change their methylation state by drought stress (Fig. 5b), of which we found that approximately $66.67 \%(4 / 6)$, $55.56 \%$ (5/9), $43.75 \%(7 / 16)$ and $41.17 \%(14 / 34)$ of genes related to ethylene, gibberellin, cytokinin and auxin were induced to be de-methylated, which was consistent with the result of the content variations of plant hormones (Fig. 5c). To further examine the regulatory roles of DNA methylation on the hormone-related genes, we conducted the expression analysis of 65 genes with qRT-PCR, and the results showed that $83.07 \%(54 / 65)$ of these genes exhibited a marked change in expression, including 41 genes that were up-regulated and 13 genes that were down-regulated (Fig. 5d), which were almost in agreement with the methylation changes in the process. Zhong et al. showed that differentially methylated cytosines (DMCs) were mainly concentrated in regions $5^{\prime}$ upstream of genes and were therefore more likely to be associated with promoter regulatory regions [11]. To determine whether the epigenetic variations were associated with promoter regions, we screened the locations of these differentially methylated cytosines (DMCs) through BLAST analysis between control and drought-treated samples. We observed the enticement of DMCs (50/65) in promoter regions, which were similar to the methylation variations reported in Arabidopsis thaliana leaves [5, 36]. Consequently, this finding might indicate that DNA methylation in the promoter regions of hormone-related genes is most likely to be involved in the regulation of plant hormones (including ethylene, auxin, gibberellin and cytokinin), which is a possible mechanism 


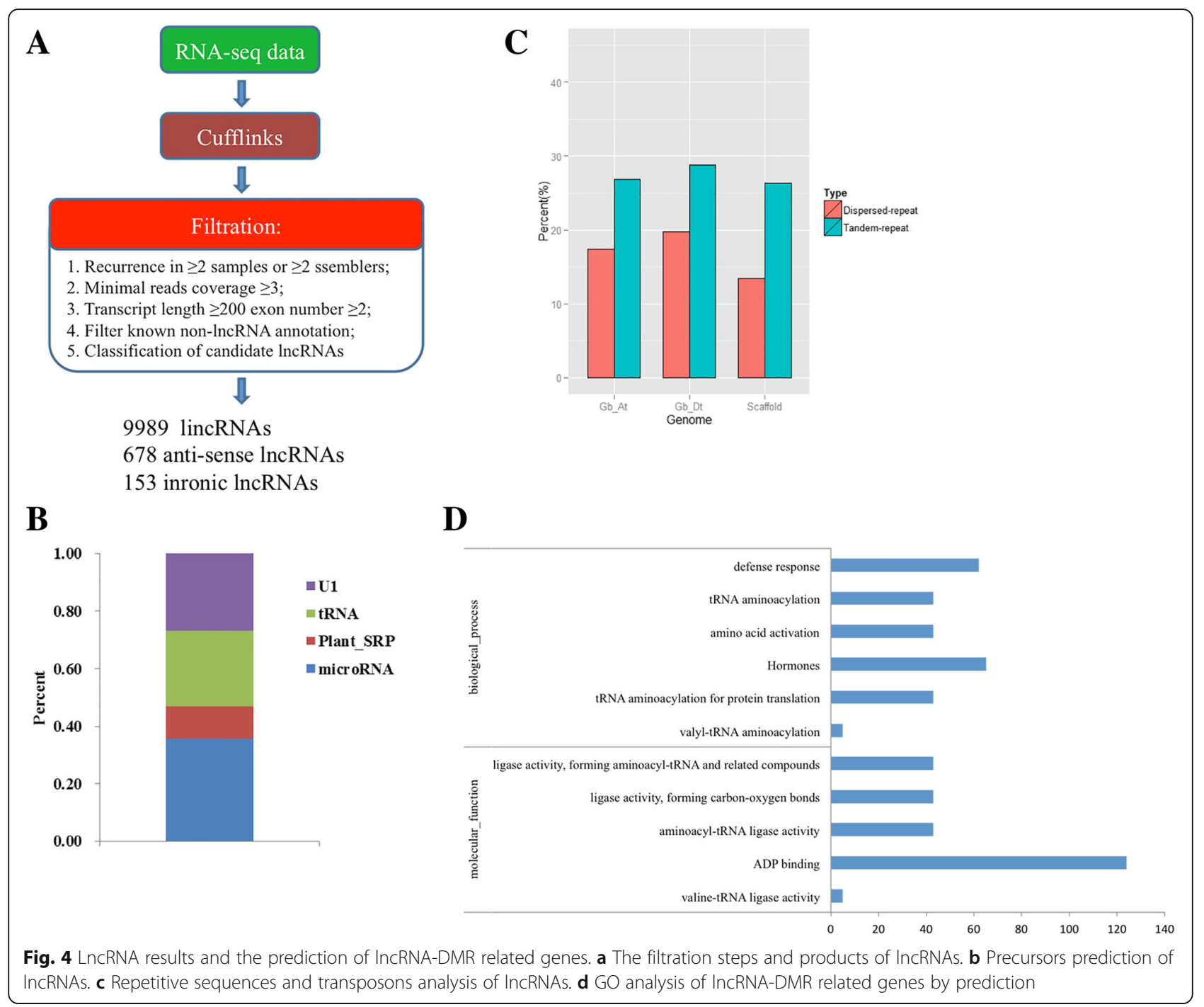

for controlling plant hormones in the drought response and may contribute to the drought resistance of cotton.

\section{Methyltransferase inhibition could enhance drought resistance}

It has been supposed that DNA methylation contributes to the response to drought stress in cotton because the alterations in methylation status would change the transcription and expression of hormone-related genes. To determine whether the methylation changes in these genes participate in regulating the response to drought (and because no methyltransferase mutants are available in cotton), we injected 5-azacytidine, a general inhibitor of DNA (mainly 5-cytosines) methyltransferases, into cotyledons before drought stress treatment and discovered improved resistance in the treated plants. The cotyledons of the 5-azacytidine-treated cotton plants were all still green, while most of the untreated or $\mathrm{dd}_{2} \mathrm{O}$-treated cotyledons turned yellow and even began to fall off under the stress (Fig. 6a). Gene expression analysis revealed the substantial decrease in the methyltransferase-related lncRNAs XLOC_181940, XLOC_005742 and XLOC_289283 and the sharp increase in their targets, CotAD_50357, CotAD_14650 and CotAD_72292, which also confirmed the reliability of the method. Furthermore, the methylation-specific PCR (MSP) results confirmed that $75.38 \%(49 / 65)$ of the hormone-related genes identified were induced to methylation changes (Fig. 6b), of which $66.7 \%(4 / 6), 77.78 \%$ (7/9), 50\% (8/16) and 52.94\% (18/ 34) of the genes related to ethylene, gibberellin, cytokinin and auxin, respectively, were de-methylated. Expression analysis showed that more genes presented an up-regulated trend by a large margin (Fig. 6c). Interestingly, one gene (named CotAD_60689) associated with auxin was discovered to be regulated through hyper- or hypo-methylation changes, and the position of the gene is shown in Fig. 6d. Next, we conducted methylation site prediction for CotAD_60689, and two sites were 

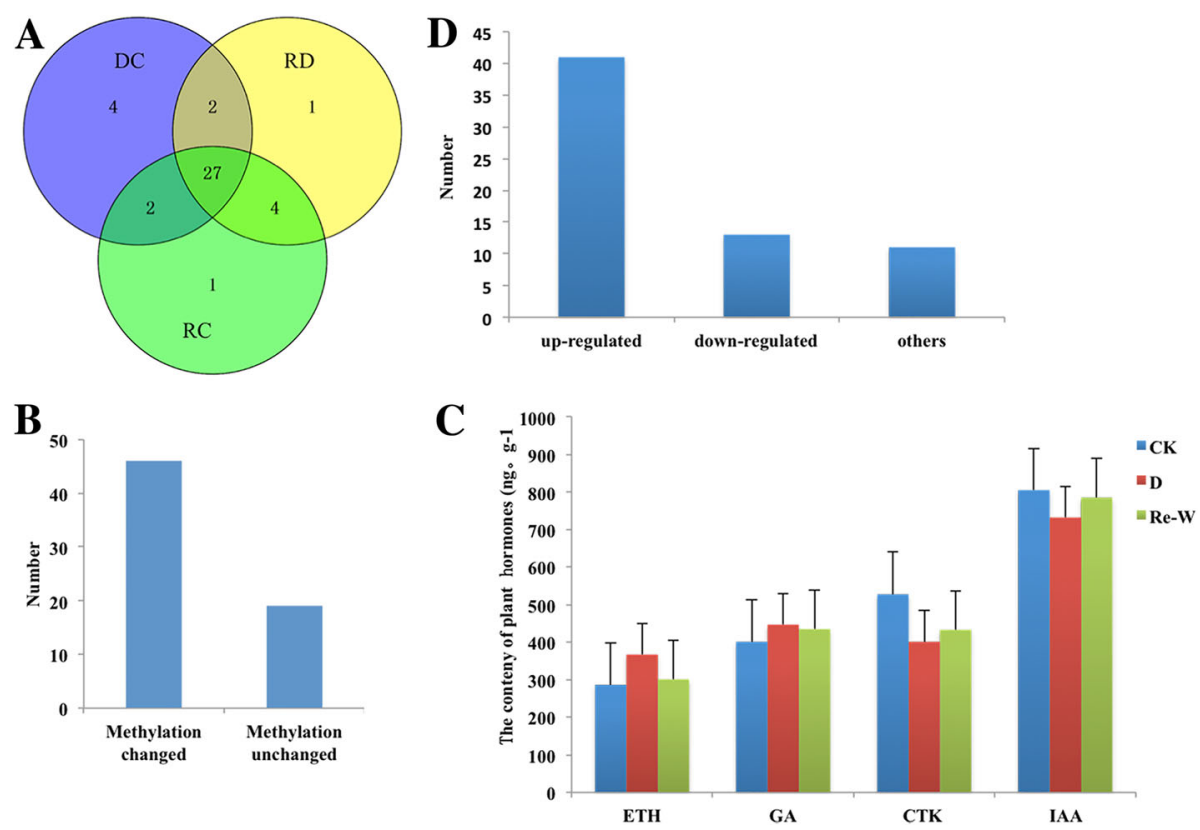

Fig. 5 Regulation of plant hormones may be associated with DNA methylation. a Venn diagram of differentially expressed genes associated with hormones. DC, RD and RC represent differentially expressed hormone genes between drought and control, re-watering and drought, and re-watering and control. $\mathbf{b}$ Number of genes with methylation-state changed. c The content variations of plant hormones under drought stress and re-watering treatment. $\mathbf{d}$ Expression analysis of plant hormone-related genes with qRT-PCR

found (site 1 was located at 686-697, and site 2 was located at 1449-1556) (Fig. 6e). Based on the observations that ? more than two-thirds (46/65) of hormone-related genes epigenetically changed under drought stress and that $\cos _{3}$ methylation inhibitor treatment could enhance the drought tolerance by delaying the speed of falling and turning yellow of cotyledons, we proposed that the inhibition of DNA methyltransferase removes the transcript and expression constraint that prevents massive expression of some hormone-related genes during the drought stress response.

\section{Discussion}

Drought stress, together with other adversities, such as salt, cold and biotic factors, generates serious challenges to plant growth and development. Hence, plants have developed remarkable capabilities to modulate the physiological and molecular machinery through genome-wide geneexpression changes in response to these environmental perturbations [37]. This study provides insights into the potential of a dynamic epigenome in the drought response in Gossypium hirsutum L. Our data showed that drought stress could induce an up-regulated epigenome, in which three sequence contexts, including ${ }^{\mathrm{m}} \mathrm{CpG},{ }^{\mathrm{m}} \mathrm{CHG},{ }^{\mathrm{m}} \mathrm{CHH}$ contexts, all showed a hyper-methylation pattern after drought stress, which was consistent with previous research [38]. Furthermore, the methylation level decreased to some extent but was still slightly higher in re-watered cotton seedlings than in the control, which suggested that some methylation variations could be retained by memory and even inherited by the next few generations and that many methylation variations changed with the treatments; when the stress was removed, these methylation sites could be restored to their original state. Thus, droughtinduced epigenetic changes in the cotton genome can be considered a very important regulatory mechanism for cotton plants to adapt to drought and possibly other environmental stresses. Moreover, more significant changes were found in asymmetric $\mathrm{CHH}$ contexts than in CG and CHG contexts, changing dynamically with the external environments, which suggested that $\mathrm{CHH}$ methylation may be mostly correlated with environments. Publications have shown that $\mathrm{CHH}$ DNA methylation/demethylation may constitute a potential novel epigenetic modification mechanism that regulates growth performance in higher plants over the stress period [39]. Thus, we deduced that methylation/demethylation in $\mathrm{CHH}$ contexts may be correlated with external environments and different growth stages, comprising a complex epigenetic regulatory pathway with other epigenetic modifications, such as histone acetylation and histone methylation.

Based on the fact that DNA methylation is always associated with nucleotide sequences [40], we found that the preference for DNA methylation is possibly associated with the symmetric sequence context of CG and CHG and sequence regions with a high or low density of DNA methylation. Many lines of evidence have proven that epigenetic modifications, such as DNA methylation and histone modification, play a crucial role in regulating 

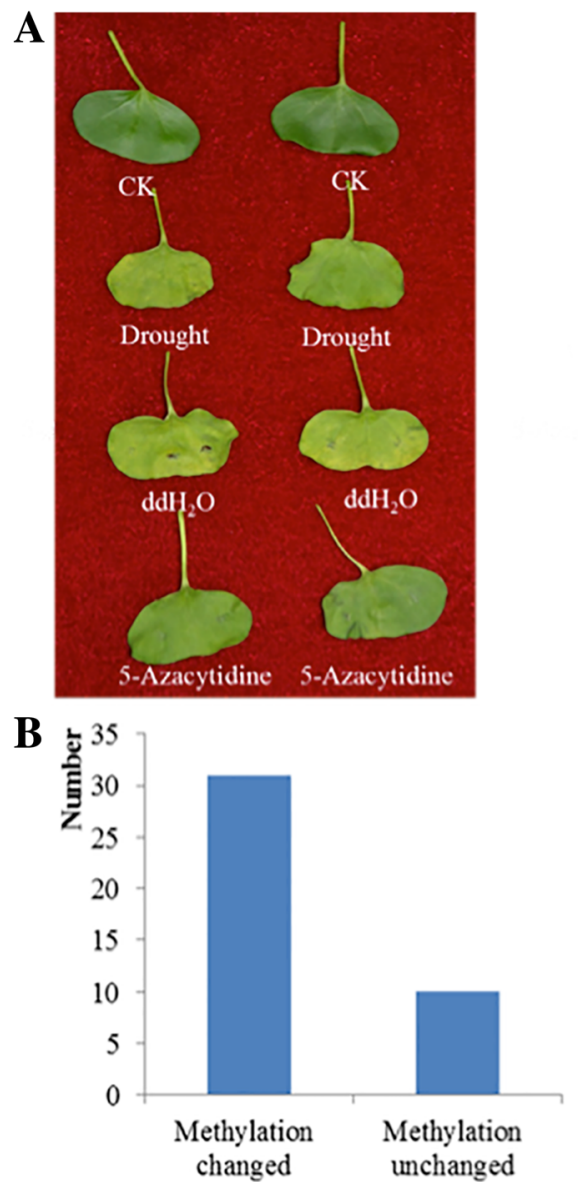

$\mathbf{C}$

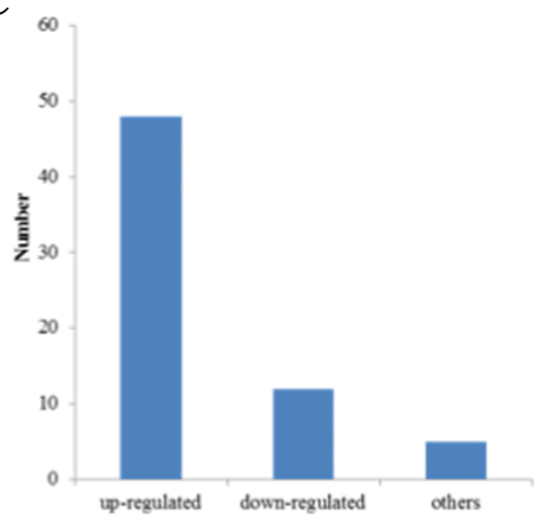

D

Scaffold829.1

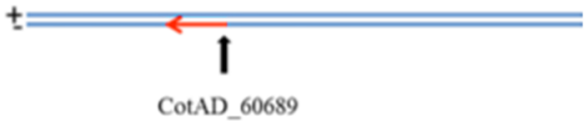

$\mathbf{E}$

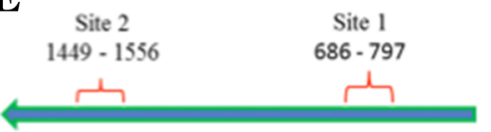

Fig. 6 Application of a methylation inhibitor to enhance drought resistance. a Morphological changes of cotyledons between CK and 5Azacytidine-treated under drought stress. CK represents cotyledons without any treatments; Drought represents cotyledons under drought stress; $\mathrm{dd}_{2} \mathrm{O}$ and 5-Azacytidine represent cotyledons injected with $\mathrm{dd}_{2} \mathrm{O}$ and 5-Azacytidine, respectively. Two cotyledons from each treatment were selected and all showed a similar symptom. b Number of genes methylation state changed of 41 hormone- related genes. c Expression analysis of plant hormone-related genes with qRT-PCR. $\mathbf{d}$ The location of two Auxin-related genes. e Prediction of methylation sites of target gene CotAD_60689. Criteria: size > 100, GC Percent > 50.0, Obs/Exp > 0.6

gene expression in response to various environmental stresses in plants [41, 42]. DNA methylation state alterations in different regions (promoters, exons and introns) cause different gene expression changes. Unlike the CG context and the $\mathrm{CHG}$ context, $\mathrm{CHH}$ context methylation in promoter regions presents hyper-methylation under drought stress and then recovers to normal levels, which shows that $\mathrm{CHH}$ context methylation dynamically changes and is most closely related to the environments. Publications have proven that methylation in the $\mathrm{CHH}$ context promotes de novo methylation on flanking intergenic chromatin (particularly within $1 \mathrm{~kb}$ of gene starts and ends) [43]. Thus, the disproportionally high levels of $\mathrm{CHH}$ relative to $\mathrm{CG}$ and $\mathrm{CHG}$ that we found in this study suggested that a skewed ratio of de novo methylation near genes would be beneficial for the drought response. Furthermore, methylation alterations in promoter regions would lead to a greater change in expression than those in other gene elements, such as exons and introns, which may be because methylation in promoter regions would influence the transcription of genes with microRNAs and RNA polymerases. Studies in humans have also shown that DNA methylation is an important epigenetic mechanism for gene silencing and cancer progression and that aberrant methylation is mainly found in CpG dinucleotides within promoter regions, which is an important pathway for the repression of gene transcription [44, 45].

On the basis of our observations that (1) the methylation status of 41 selected hormone-related genes changed according to the external environment (ck, drought and re-watering), (2) the hormone content and gene expression level of these 41 genes also changed with treatment by drought and re-watering, and (3) methylation inhibitor injection induced the enhancement of drought tolerance and the alterations of methylation status, we proposed that the methylation status of hormone- 
related genes is one of the mechanisms responding to drought stress. Studies have shown that hormones (proline, ABA, ZR, ETH and AUX, etc.) are associated with drought tolerance and that selecting and using cultivars with a higher proline, ABA, ZR and AUX content under drought stress would be a practical approach to improve drought tolerance in plants [46]. Expression analysis of these 41 hormone-related genes in the investigation also confirmed these results. Combined with the expression data of these hormone-related genes, the long non-coding RNAs and microRNAs, we inferred that long non-coding RNAs would regulate the occurrence of methylation by splicing into microRNAs (RNA-directed DNA methylation, RdDM) in hormone content regulation in response to drought stress. Karakulah et al. have reported that both lncRNAs and microRNAs could block the binding of specific transcript leading to increase mRNA expression by a newly discovered regulatory mechanism called endogenous target mimicry (eTM) [47]. Based on our results and those of previous studies, we propose a four-component model for hormone regulation, in which, through interacting mechanisms that remain unclear, long non-coding RNAs can direct DNA methylation through splicing into microRNAs in response to drought stress (Fig. 7). Angiosperms seem to use their epigenomic stability as a supplemental regulatory mechanism controlling a developmental transition that could cause severe energy and substance waste if it were to transit from normal growth to response to various stresses.

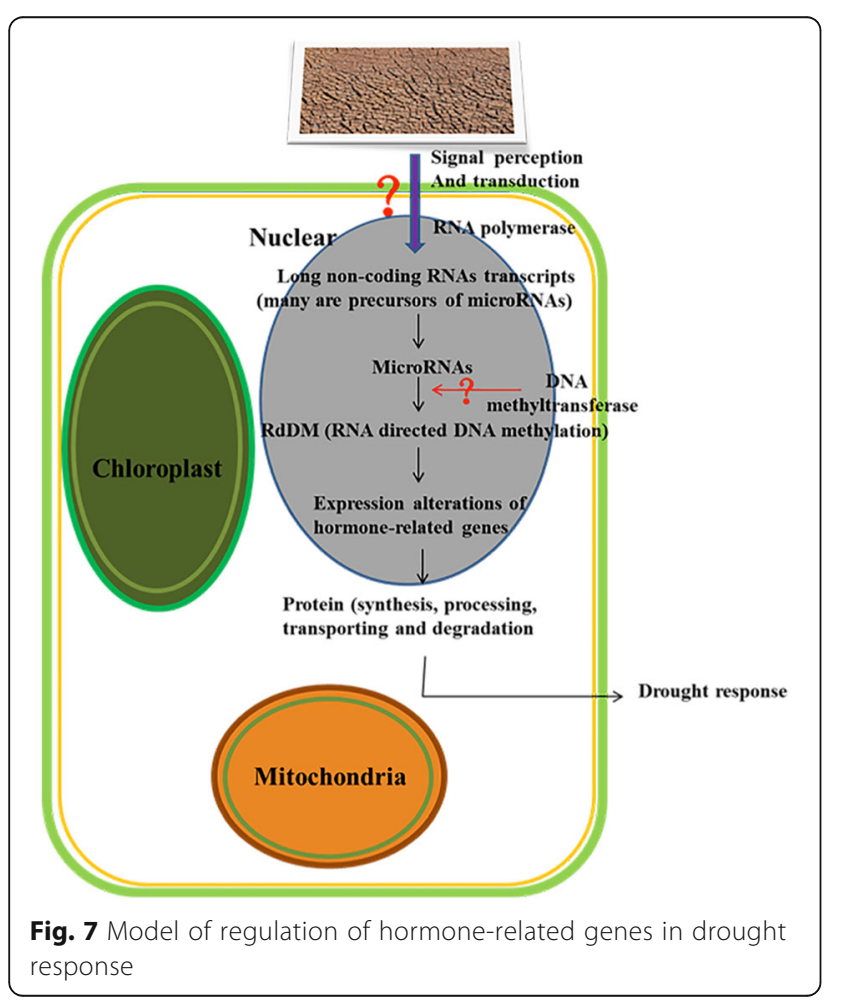

\section{Conclusions}

This study provides insights into the regulatory network of the dynamic epigenome in response to drought. In addition, previous searches for the "cryptic regulatory role" that promotes responding to stresses and is of significant agricultural value were focused on the expression of stress-tolerance genes, stress-related molecular markers and hormone signaling [48-51]. Indeed, plant breeding projects depend on DNA-base molecular markers (such as QTLs and SNPs) and may overlook much epigenetic variation. Hence, our studies highlight the significance of epigenetic modifications, which are beneficial for future crop-improvement strategies and technologies that consider not only genetic variations but also epigenetic modifications. During the process of responding to drought stress, the DMRs associated with drought tolerance, as well as the hormone-related genes and transcription factors described here, provide an initial set of targets for analyzing epigenomic variations across the identified drought-tolerant cotton varieties and for assessing variations that may form the basis of future expanded selection strategies. Moreover, other pathways, for example, metabolic pathways, signal transduction and ripening, may involve methylation variations, which will require additional experimental data for confirmation.

\section{Methods}

\section{Sample collection and preparation}

Drought-tolerant upland cotton (Gossypium hirsutum L.) ZhongH177 seeds were preserved in the Cotton adversity research laboratory (our laboratory) in Institute of Cotton Research of Chinese Academy of Agricultural Sciences for many years. The seeds were sterilized with $0.1 \% \mathrm{HgCl}_{2}$ and placed in a sterile culture dish to accelerate germination before planting into the sand. Cotton seedlings with uniform size were planted into a sand container (10 seedlings per container) in the greenhouse $\left(14 \mathrm{~h} /\right.$ day, $30^{\circ} \mathrm{C}$ and $10 \mathrm{~h} /$ night, $24{ }^{\circ} \mathrm{C}$ ) of the Institute of Cotton Research of Chinese Academy of Agricultural Sciences. At trefoil stage (three true leaves), drought stress was conducted by withholding watering till the relative water content (RWC) in pots reaches to about $7.0 \%$, while the control pots were watered as before. For methyltransferase inhibitor treatment, $500 \mu \mathrm{l}$ (a small part of which would be wasted in the injection process) of $1 \mathrm{mM} 5$-azacytidine aqueous solution was injected into the cotyledons. Then the second and third true leaves from ten plants were harvested together, snap frozen with liquid nitrogen, and stored at $-80{ }^{\circ} \mathrm{C}$ until use. The method used for sampling was the same for both the control and treatment samples. And the long non-coding RNA sequencing was repeated with three times and whole-genome bisulfite sequencing was conducted with the sequencing depth was $30 \times$. 
DNA, RNA isolation, quantification and qualification Genomic DNA and RNA were extracted with CTAB method [52]. Genomic DNA and RNA degradation and contamination were monitored on $1 \%$ agarose gels. DNA and RNA purity were checked using the NanoPhotometer spectrophotometer (IMPLEN, CA, USA). DNA concentration was measured using Qubit DNA Assay Kit in Qubit 2.0 Flurometer (Life Technologies, CA, USA).

\section{Library preparation and quantification}

A total amount of 5.2 microgram genomic DNA spiked with $26 \mathrm{ng}$ lambda DNA were fragmented by sonication to 200-300 bp with Covaris S220, followed by end repair and adenylation. Cytosine-methylated barcodes were ligated to sonicated DNA as per manufacturer's instructions. Then these DNA fragments were treated twice with bisulfite using EZ DNA Methylation-Gold Kit (Zymo Research). And the resulting single-strand DNA fragments were PCR amplificated using KAPA HiFi HotStart Uracil + Ready Mix (2X). Library concentration was quantified by Qubit 2.0 Flurometer (Life Technologies, CA, USA) and quantitative PCR, and the insert size was checked on Agilent Bio-analyzer 2100 system. The method of lncRNAs library construction, sequencing, data analysis were shown as Lu et al. [53].

\section{Clustering and sequencing}

The clustering of the index-coded samples was performed on a cBot Cluster Generation System using TruSeq PE Cluster Kit v3-cBot-HS (Illumia) according to the manufacturer's instructions. After cluster generation, the library preparations were sequenced on an Illumina Hiseq 2000/2500 platform and $100 \mathrm{bp} / 50 \mathrm{bp}$ single-end reads were generated. Image analysis and base calling were performed with the standard Illumina pipeline, and finally $100 \mathrm{bp}$ paired-end reads were generated.

\section{Data analysis \\ Quality control}

Read sequences produced by the Illumina pipeline in FastQ format were first pre-processed through in-house perl scripts. Firstly, as a subset of reads contained all of part of the 3'adapter oligonucleotide sequence, every read was scanned for the adapter sequence, and if detected the read was filtered out. Secondly, since some reads had N (unknown base) in their sequences, the percentage of Ns in each read was calculated, and if the percentage of Ns was larger than $10 \%$ the read was removed. Thirdly, reads with low quality (PHRED score $\leq 5$, and percentage of the low quality bases $\geq 50 \%$ ) were trimmed. At the same time, Q20, Q30andGC content of the data were calculated. The remaining reads that passed the filters were called as clean reads and all of the subsequent analyses were based on them.

\section{Reads mapping to the reference genome}

Bismark software (version 0.12.5) [54] was used to perform alignments of bisulfite-treated reads to a reference genome with the default parameters. The reference genome was firstly transformed into bisulfite-converted version (Cto-T and G-to-A converted) and then indexed using bowtie2 [55]. Sequence reads were also transformed into fully bisulfite-converted versions (C-to-T and G-to-A converted) before they are aligned to similarly converted versions of the genome in a directional manner. Sequence reads that produce a unique best alignment from the two alignment processes (original top and bottom strand) are then compared to the normal genomic sequence and the methylation state of all cytosine positions in the read is inferred. The same reads that aligned to the same regions of genome were regarded as duplicated ones. The sequencing depth and coverage were summarized using deduplicated reads. The results of methylation extractor were transformed into bigWig format for visualization using IGV browser. The sodium bisulfite non-coversion rate was calculated as the percentage of cytosines sequenced at cytosine reference positions in the lambda genome.

\section{Estimating methylation level}

To identify the methylation site, we modeled the sum $S_{i, j}^{+}$ of methylated counts as a binomial (Bin) random variable with methylation rate $r_{i, j}$

$$
S^{+}{ }_{i, j} \sim \operatorname{Bin}\left(S^{+}{ }_{i, j}+S^{-}{ }_{i, j}, r_{i, j}\right)
$$

We employed a sliding-window approach, which is conceptually similar to approaches that have been used for bulk BS-Seq (http://www.bioconductor.org/packages/ $2.13 / \mathrm{bioc} / \mathrm{html} / \mathrm{bsseq} \cdot \mathrm{html}$ ). With window size $\mathrm{w}=$ 3,000 bp and step size 600 bp [56], the sum of methylated and unmethylated read counts in each window were calculated. Methylation level (ML) for each C site shows the fraction of methylated $\mathrm{Cs}$, and is defined as:

$$
M L(C)=\frac{\operatorname{reads}(m C)}{\text { reads }(m C)+\text { reads }(C)}
$$

Calculated ML was further corrected with the bisulfite non-conversion rate according to previous studies [57]. Given the bisulfite non-conversion rate $r$, the corrected ML was estimated as:

$$
M L(\text { corrected })=\frac{M L-r}{1-r}
$$

\section{Differentially methylated regions analysis}

Differentially methylated regions (DMRs) were identified using the swDMR software (http://122.228.158.106/ swDMR/), which uses a sliding-window approach. The 
window is setto $1000 \mathrm{bp}$ and step length is $100 \mathrm{bp}$. Fisher test is implemented to detect the DMRs.

\section{GO and KEGG enrichment analysis of DMR-related genes} Gene Ontology (GO) enrichment analysis of genes related to DMRs was implemented by the GO-seq R package [58], in which gene length bias was corrected. GO terms with corrected P-value less than 0.05 were considered significantly enriched by DMR-related genes. KEGG [59] is a database resource for understanding high-level functions and utilities of the biological system, such as the cell, the organism and the ecosystem, from molecular-level information, especially large-scale molecular datasets generated by genome sequencing and other high-through put experimental technologies (http://www.genome.jp/kegg/). We used KOBAS software [60] to test the statistical enrichment of DMR-related genes in KEGG pathways.

\section{MicroRNA prediction analysis}

Based on the 10,820 long non-coding RNAs, we conducted microRNA prediction analysis with rfam-scan program. Besides, cmsearch program was also used to search homologous microRNAs in database rfam11, and the screening threshold value was $\mathrm{E}$ value $<=1 \mathrm{E}-05$. And the method used here was the same as the description by Lu et al [49].

\section{5 - Azacytidine treatment}

At trefoil stage, 5-azac was diluted with $\mathrm{dd}_{2} \mathrm{O}$ to $5 \mathrm{mg} \cdot \mathrm{ml}^{-1}$. The diluted 5 -azaC was injected into cotton true leaves with an injection syringe, as the method described by Zhong. et al [11], while the blank control and negative control were performed with nothing and equal amount $\mathrm{ddH}_{2} \mathrm{O}$, respectively. The injection work was ceased when the liquid spread to $90 \%$ area of the leaves. The samples, both the controls and treatment, were harvested when the leaves turned yellow (nearly $24 \mathrm{~h})$.

\section{Methylation specific PCR (MSP)}

Before carrying out MSP test, DNA bisulfite conversion should be done with DNA bisulfite conversion kit (TIANGEN). In which, Bisulfite mix should be prepared firstly and a $120 \mu \mathrm{l}$ volume system, containing DNA $3.3 \mu \mathrm{l}(\sim 300 \mathrm{ng} \bullet \mu \mathrm{l}-1)$, Bisulfite mix $90 \mu \mathrm{l}$ and $\mathrm{ddd}_{2} \mathrm{O}$ $26.7 \mu \mathrm{l}$, was used in the conversion process. After the preparation, the program used in the reaction was $95{ }^{\circ} \mathrm{C}$, $10 \mathrm{~min}$, then $64^{\circ} \mathrm{C}, 60 \mathrm{~min}$, then $4{ }^{\circ} \mathrm{C}$, forever. If DNA content was $<500 \mathrm{ng}$, time could be shorten to $30 \mathrm{~min}$ at $64{ }^{\circ} \mathrm{C}$, treatment would be all right. If DNA content was $>500 \mathrm{ng}, 60 \mathrm{~min}$ should be used. After the bisulfite conversion, bisulfite-treated DNA should be purified as the direction.

Purified bisulfite-treated DNA was used as templates for methylation specific PCR (MSP), and MSP primers were designed with online program (http://www.urogene.org/ cgi-bin/methprimer/methprimer.cgi) [61]. $20 \mu \mathrm{l}$ volume system, containing template DNA $3.5 \mu \mathrm{l}(\sim 100 \mathrm{ng} \bullet \mu \mathrm{l}-1)$, forward primer $1 \mu \mathrm{l}(10 \mu \mathrm{M})$, reverse primer $1 \mu \mathrm{l}(10 \mu \mathrm{M})$, dNTPs $1.6 \mu \mathrm{l}(2.5 \mathrm{mM})$, MSP DNA polymerase $0.5 \mu \mathrm{l}(2.5$ $\mathrm{U} \cdot \mu \mathrm{l}-1), 10 \times \mathrm{PCR}$ buffer $2 \mu \mathrm{l}$ and ddH2O $10.3 \mu \mathrm{l}$ was used. Program in the MSP reaction was $95{ }^{\circ} \mathrm{C}, 5 \mathrm{~min}, 94{ }^{\circ} \mathrm{C}, 20 \mathrm{~s}$, $60{ }^{\circ} \mathrm{C}, 30 \mathrm{~s}, 72{ }^{\circ} \mathrm{C}, 20 \mathrm{~s}, 72{ }^{\circ} \mathrm{C}, 5 \mathrm{~min}, 35$ cycles. After the MSP reaction, $10 \mu \mathrm{l}$ PCR production was used to detection by $1 \%$ agarose gel electrophoresis.

\section{Hormone content determination}

Plant hormones content determination method was shown as Lu et al [53].

\section{Additional files}

Additional file 1: Figure S1. Distribution of methylation sites in each chromosome in control sample (PNG $1360 \mathrm{~kb}$ )

Additional file 2: Figure S2. Distribution of methylation sites in each chromosome in drought-treated sample (PNG $1385 \mathrm{~kb}$ )

Additional file 3: Figure S3. Distribution of methylation sites in each chromosome in re-watered sample3 (PNG 1367 kb)

Additional file 4: Figure S4. DMRs length analysis in each chromosome between drought and control sample (PNG 107 kb)

Additional file 5: Figure S5. DMRs length analysis in each chromosome between re-watering and drought sample (PNG 103 kb)

Additional file 6: Figure S6. DMRs length analysis in each chromosome between re-watering and control sample (PNG 108 kb)

Additional file 7: Figure S7. GO analysis of hyper-methylated genes associated with drought (PNG $111 \mathrm{~kb}$ )

Additional file 8: Figure S8. GO analysis of hypo-methylated genes associated with drought (PNG $102 \mathrm{~kb}$ )

Additional file 9: Figure S9. Statistics of Pathway Enrichment of differentially methylated genes (PDF $6 \mathrm{~kb}$ )

\section{Abbreviations}

DMCs: Differentially methylated cytosines; DMRs: Differentially methylated regions; eTM: Endogenous target mimicry; LncRNAs: Long non-coding RNAs; MSP: Methylation-specific PCR; NcRNAs: Non-coding RNAs; RdDM: RNA-directed DNA methylation; RWC: Relative water content; WGBS: Whole-genome bisulfite sequencing

\section{Acknowledgments}

We thank Ruifeng Cui and Binglei Zhang for the help with the preparation of the materials, and Mingge Han and Qi Li and other members in our group for the help with the experiments. We also thank for the members involved in the project in Novogene (Beijing) for the help with whole-genome bisulfite sequencing and data analysis. We also thank reviewers for checking our manuscript and the editors for editing the paper. All authors have declared that no competing interests exist.

\section{Funding}

This project was supported by grants from the National $13^{\text {th }}$ Five-Year major science and technology projects (Functional genomes and Networks of fibre quality and adversity-resistance in cotton: 2016YFD0101006).

\section{Availability of data and materials}

All analysis results data generated during this study are included in this article and its supplementary information. The raw data from this study was uploaded to BioSample database accession ID SRX2012512 (released until 2019-01-01). 


\section{Authors' contributions}

XKL and WWY designed the experiments and wrote manuscript. XGW, XGC, NS, JJW and DLW helped the experiments. SW, WLF and LXG managed the materials in the field. All authors read and approved the final manuscript.

\section{Competing interests}

The authors declare that they have no competing interests.

\section{Consent for publication}

Not applicable.

\section{Ethics approval and consent to participate}

All the cotton materials were collected from the Institute of Cotton Research, Chinese Academy of Agricultural Sciences, which are public and available for non- commercial purpose.

\section{Publisher's Note}

Springer Nature remains neutral with regard to jurisdictional claims in published maps and institutional affiliations.

\section{Author details}

${ }^{1}$ State Key Laboratory of Cotton Biology/Institute of Cotton Research of Chinese Academy of Agricultural Sciences/Key Laboratory for Cotton Genetic Improvement, Anyang 455000, Henan, China. ${ }^{2}$ College of Agronomy, Xinjiang Agricultural University, Urumqi 830052, China.

Received: 6 December 2016 Accepted: 5 April 2017

Published online: 13 April 2017

\section{References}

1. Cubas $P$, Vincent $C$, Coen $E$. An epigenetic mutation responsible for natural variation in floral symmetry. Nature. 1999:401:157-61. doi:10.1038/43657.

2. Manning $\mathrm{K}$, et al. A naturally occurring epigenetic mutation in a gene encoding an SBP-box transcription factor inhibits tomato fruit ripening. Nat Genet. 2006;38:948-52. doi:10.1038/ng1841.

3. Cokus SJ, et al. Shotgun bisulphite sequencing of the Arabidopsis genome reveals DNA methylation patterning. Nature. 2008;452:215-9. doi:10.1038/ nature06745.

4. Lister $\mathrm{R}$, et al. Highly integrated single-base resolution maps of the epigenome in Arabidopsis. Cell. 2008;133:523-36. doi:10.1016/j.cell.2008.03.029.

5. Becker $C$, et al. Spontaneous epigenetic variation in the Arabidopsis thaliana methylome. Nature. 2011;480:245-U127. doi:10.1038/nature10555.

6. Paterson $\mathrm{AH}$, et al. Repeated polyploidization of Gossypium genomes and the evolution of spinnable cotton fibres. Nature. 2012:492:423. doi:10.1038/ nature11798.

7. Naoumkina M, Hinchliffe DJ, Turley RB, Bland JM, Fang DD. Integrated metabolomics and genomics analysis provides new insights into the fiber elongation process in Ligon lintless-2 mutant cotton (Gossypium hirsutum L.). BMC Genomics. 2013;14:155. doi:10.1186/1471-2164-14-155.

8. Li F, et al. Genome sequence of the cultivated cotton Gossypium arboreum Nat Genet. 2014;46:567-72. doi:10.1038/ng.2987.

9. Wang K, et al. The draft genome of a diploid cotton Gossypium raimondii. Nat Genet. 2012;44:1098-103. doi:10.1038/ng.2371.

10. Li F, et al. Genome sequence of cultivated Upland cotton (Gossypium hirsutum TM-1) provides insights into genome evolution. Nat Biotechnol. 2015;33:524-30. doi:10.1038/nbt.3208.

11. Zhong SL, et al. Single-base resolution methylomes of tomato fruit development reveal epigenome modifications associated with ripening. Nat Biotechnol. 2013; 31:154-9. doi:10.1038/nbt.2462

12. Zemach $A$, et al. Local DNA hypomethylation activates genes in rice endosperm. P Natl Acad Sci USA. 2010;107:18729-34. doi:10.1073/pnas. 1009695107

13. Wang WS, et al. Drought-induced site-specific DNA methylation and its association with drought tolerance in rice (Oryza sativa L.). J Exp Bot. 2011; 62:1951-60. doi:10.1093/jxb/erq391.

14. Liang D, et al. Single-base-resolution methylomes of Populus trichocarpa reveal the association between DNA methylation and drought stress. BMC Genet. 2014;15 Suppl 1:S9. doi:10.1186/1471-2156-15-S1-S9.

15. Wang B, et al. Analysis of methylation-sensitive amplified polymorphism in different cotton accessions under salt stress based on capillary electrophoresis. Genes Genom. 2015;37:713-24. doi:10.1007/s13258-015-0301-6.
16. Wan $P$, et al. Computational analysis of drought stress-associated miRNAs and miRNA co-regulation network in Physcomitrella patens. Genomics Proteomics Bioinformatics. 2011;9:37-44. doi:10.1016/S1672-0229(11)60006-5.

17. Chen $\mathrm{Q}$, et al. Integrated mRNA and microRNA analysis identifies genes and small miRNA molecules associated with transcriptional and post-transcriptionallevel responses to both drought stress and re-watering treatment in tobacco. BMC Genomics. 2017;18:62. doi:10.1186/s12864-016-3372-0.

18. Payton $P$, et al. Examining the drought stress transcriptome in cotton leaf and root tissue. Biotechnol Lett. 2011;33:821-8. doi:10.1007/s10529-010-0499-y.

19. Zhou JC, et al. H19 IncRNA alters DNA methylation genome wide by regulating S-adenosylhomocysteine hydrolase. Nature communications. 2015; 6. doi:Artn 1022110.1038/Ncomms10221.

20. Di Ruscio A, et al. DNMT1-interacting RNAs block gene-specific DNA methylation. Nature. 2013:503:371. doi:10.1038/nature12598.

21. Richards EJ. Natural epigenetic variation in plant species: a view from the field. Curr Opin Plant Biol. 2011;14:204-9. doi:10.1016/j.pbi.2011.03.009.

22. Harris RA, et al. Comparison of sequencing-based methods to profile DNA methylation and identification of monoallelic epigenetic modifications. Nat Biotechnol. 2010;28:1097-U1194. doi:10.1038/nbt.1682.

23. Chen PY, Feng S, Joo JW, Jacobsen SE, Pellegrini M. A comparative analysis of DNA methylation across human embryonic stem cell lines. Genome Biol. 2011;12:R62. doi:10.1186/gb-2011-12-7-r62.

24. Lister $\mathrm{R}$, et al. Human DNA methylomes at base resolution show widespread epigenomic differences. Nature. 2009;462:315-22. doi:10.1038/nature08514.

25. Bedre R, et al. Genome-Wide Transcriptome Analysis of Cotton (Gossypium hirsutum L.) Identifies Candidate Gene Signatures in Response to Aflatoxin Producing Fungus Aspergillus flavus. Plos One. 2015;10. doi:10.1371/journal. pone.0138025.

26. Eprintsev AT, et al. The role of promoter methylation in the regulation of genes encoding succinate dehydrogenase in maize seedlings. Russ J Plant Physl. 2012;59:299-306. doi:10.1134/S1021443712030053.

27. Gent Jl, et al. CHH islands: de novo DNA methylation in near-gene chromatin regulation in maize. Genome Res. 2013;23:628-37. doi:10.1101/gr.146985.112.

28. Yang HX, et al. Whole-genome DNA methylation patterns and complex associations with gene structure and expression during flower development in Arabidopsis. Plant J. 2015;81:268-81. doi:10.1111/tpj.12726.

29. Holliday R, Pugh JE. DNA modification mechanisms and gene activity during development. Science. 1975;187:226-32.

30. Riggs AD. X inactivation, differentiation, and DNA methylation. Cytogenet Cell Genet. 1975;14:9-25.

31. Lai F, Shiekhattar R. Where long noncoding RNAs meet DNA methylation. Cell Res. 2014;24:263-4. doi:10.1038/cr.2014.13.

32. Goff LA, Rinn JL. Linking RNA biology to IncRNAs. Genome Res. 2015;25: 1456-65. doi:10.1101/gr.191122.115.

33. Movahedi A, et al. RNA-directed DNA methylation in plants. Plant Cell Rep. 2015;34:1857-62. doi:10.1007/s00299-015-1839-0.

34. Zhang H, He XJ, Zhu JK. RNA-directed DNA methylation in plants Where to start? RNA Biol. 2013:10:1593-6. doi:10.4161/rna.26312.

35. Castel SE, Martienssen RA. RNA interference in the nucleus: roles for small RNAs in transcription, epigenetics and beyond. Nat Rev Genet. 2013;14:100-12. doi:10.1038/nrg3355.

36. Schmitz RJ, et al. Transgenerational epigenetic instability is a source of novel methylation variants. Science. 2011;334:369-73. doi:10.1126/science. 1212959.

37. Wang W, et al. Genome-Wide Differences in DNA Methylation Changes in Two Contrasting Rice Genotypes in Response to Drought Conditions. Frontiers in plant science. 2016;7:1675. doi:10.3389/fpls.2016.01675.

38. Zhou JL, et al. Global genome expression analysis of rice in response to drought and high-salinity stresses in shoot, flag leaf, and panicle. Plant Mol Biol. 2007:63:591-608. doi:10.1007/s11103-006-9111-1.

39. Jin X, et al. A Potential Role for CHH DNA Methylation in Cotton Fiber Growth Patterns. Plos One. 2013; 8. doi:ARTN e60547 10.1371/journal.pone.0060547.

40. Bock $C$, et al. CpG island methylation in human lymphocytes is highly correlated with DNA sequence, repeats, and predicted DNA structure. Plos Genet. 2006;2:e26. doi:10.1371/journal.pgen.0020026.

41. Boyko A, et al. Transgenerational changes in the genome stability and methylation in pathogen-infected plants: (virus-induced plant genome instability). Nucleic Acids Res. 2007;35:1714-25. doi:10.1093/nar/gkm029.

42. Boyko A, Kovalchuk I. Epigenetic control of plant stress response. Environ Mol Mutagen. 2008;49:61-72. doi:10.1002/em.20347. 
43. Ng DW, et al. A Role for $\mathrm{CHH}$ Methylation in the Parent-of-Origin Effect on Altered Circadian Rhythms and Biomass Heterosis in Arabidopsis Intraspecific Hybrids. Plant Cell. 2014;26:2430-40. doi:10.1105/tpc.113.115980.

44. Herman JG, Baylin SB. Mechanisms of disease: Gene silencing in cancer in association with promoter hypermethylation. New Engl J Med. 2003;349: 2042-54. doi:10.1056/Nejmra023075.

45. Hua XM, et al. DNA methylation level of promoter region of activating transcription factor 5 in glioma. J Zhejiang Univ-Sc B. 2015;16:757-62. doi: 10.1631/jzus.B1500067.

46. Man D, Bao YX, Han LB, Zhang XZ. Drought tolerance associated with proline and hormone metabolism in two tall fescue cultivars. Hortscience. 2011:46:1027-32.

47. Karakulah G, Yucebilgili Kurtoglu K, Unver T. PeTMbase: a database of plant endogenous target mimics (eTMs). Plos One. 2016;11:e0167698. doi:10.1371/ journal.pone.0167698.

48. Park W, Scheffler BE, Bauer PJ \& Campbell BT. Genome-wide identification of differentially expressed genes under water deficit stress in upland cotton (Gossypium hirsutum L.). BMC Plant Biol. 2012; 12. doi:Artn 9010.1186/14712229-12-90.

49. Wang $X$, et al. Mining and analysis of SNP in response to salinity stress in upland cotton (Gossypium hirsutum L.). Plos One. 2016;11:e0158142. doi:10.1371/journal.pone.0158142.

50. Liu JX, Srivastava R, Che P, Howell SH. Salt stress signaling in Arabidopsis thaliana involves a membrane-bound transcription factor AtbZIP17 as a signal transducer. Plant Signal Behav. 2008;3:56-7.

51. Liu JX, Srivastava R, Che P, Howell SH. Salt stress responses in Arabidopsis utilize a signal transduction pathway related to endoplasmic reticulum stress signaling. Plant J. 2007;51:897-909. doi:10.1111/j.1365-313X.2007. 03195.x.

52. Zhao $L$, et al. An improved $C T A B$-ammonium acetate method for total RNA isolation from cotton. Phytochem Anal. 2012;23:647-50. doi:10.1002/pca.2368.

53. Lu X, et al. Genome-wide analysis of long noncoding RNAs and their responses to drought stress in cotton (Gossypium hirsutum L.). Plos One. 2016;11:e0156723. doi:10.1371/journal.pone.0156723.

54. Krueger F, Andrews SR. Bismark: a flexible aligner and methylation caller for Bisulfite-Seq applications. Bioinformatics. 2011;27:1571-2. doi:10.1093/ bioinformatics/btr167.

55. Langmead B, Salzberg SL. Fast gapped-read alignment with bowtie 2. Nat Methods. 2012:9:357-U354. doi:10.1038/Nmeth.1923.

56. Smallwood SA, et al. Single-cell genome-wide bisulfite sequencing for assessing epigenetic heterogeneity. Nat Methods. 2014;11:817-20. doi:10. 1038/Nmeth.3035.

57. Lister R, et al. Global Epigenomic Reconfiguration During Mammalian Brain Development. 2013: 341: 629 - +. doi:Artn 1237905 10.1126/Science.1237905.

58. Young MD, Wakefield MJ, Smyth GK \& Oshlack A. Gene ontology analysis for RNA-seq: accounting for selection bias. Genome Biol. 2010; 11. doi:Arth R1410.1186/Gb-2010-11-2-R14.

59. Kanehisa $\mathrm{M}$, et al. KEGG for linking genomes to life and the environment. Nucleic Acids Res. 2008;36:D480-4. doi:10.1093/nar/gkm882.

60. Mao XZ, Cai T, Olyarchuk JG, Wei LP. Automated genome annotation and pathway identification using the KEGG Orthology (KO) as a controlled vocabulary. Bioinformatics. 2005;21:3787-93. doi:10.1093/bioinformatics/bti430.

61. Li LC, Dahiya R. MethPrimer: designing primers for methylation PCRs. Bioinformatics. 2002;18:1427-31. doi:10.1093/bioinformatics/18.11.1427.

\section{Submit your next manuscript to BioMed Central and we will help you at every step:}

- We accept pre-submission inquiries

- Our selector tool helps you to find the most relevant journal

- We provide round the clock customer support

- Convenient online submission

- Thorough peer review

- Inclusion in PubMed and all major indexing services

- Maximum visibility for your research

Submit your manuscript at www.biomedcentral.com/submit

) Biomed Central 